\title{
Adicción a internet, resiliencia y rendimiento escolar en adolescentes de Pucallpa
}

\author{
Internet addiction, resilience and school performance in adolescents of Pucallpa
}

\author{
Elsa M. Sánchez-Yarmas ${ }^{1}$
}

\section{Resumen}

La presente investigación tuvo como propósito analizar la relación entre la adicción a internet, la resiliencia y el rendimiento escolar en adolescentes de los distritos de Yarinacocha y Callería, ciudad de Pucallpa, en la región Ucayali (Perú). Esta región se caracteriza por concentrar la población de adolescentes con los niveles más bajos en rendimiento académico y que no estudian ni trabajan. El estudio es descriptivo, correlacional y comparativo. La muestra de estudio fue de 519 estudiantes de tercero, cuarto y quinto de secundaria, en edades de 14 a 18 años. Los instrumentos fueron la Escala de Resiliencia de Wagnild y Young, adaptada por Novella (2002), el Test de Adicción a Internet de Young, adaptado por Matalinares et al. (2012) y las calificaciones, brindadas por las instituciones educativas. Se hallaron correlaciones positivas pero bajas entre adicción a internet y resiliencia, y correlaciones negativas bajas entre adicción a internet y rendimiento, con tamaños de efecto pequeños o insignificantes. Se concluye que la resiliencia no parece ser un factor importante en el desempeño académico en este grupo y que es necesario desarrollar estudios posteriores en adolescentes que están fuera del sistema educativo a fin de contrastar con los presentes hallazgos.

Palabras clave: adicción a internet, resiliencia, rendimiento escolar, escolares

\begin{abstract}
This research had as purpose to analyze the relationship between internet adicction, resilience and school performance in adolescents from the districts of Yarinacohca and Calleria, Pucallpa city, in the región of Ucayali (Peru). This region has as characteristics to concentrate the population of adolescents with one of the lowest school performance in the country and who do neither study nor work. This study was controlled correlational and comparative. The sample was composed by 519 high school students (third, fourth and fifth grade), from 14 to 18 years old. The instruments were the Wagnild and Young's Resilience Scale (adapted by Novella, 2002) and the Young's Internet Addiction Test (adapted by Matalinares et al., 2012). Schools provided califications of students. Low positive correlations between internet addiction and resilience and low negative correlations between addiction and performance were found, with small or insignificant effect sizes. It is concluded that resilience does not seem an important factor in the school performance in this group and it is necessary to carry out further research in adolescents who are out from school to contrast with these findings.
\end{abstract}

Key words: internet addiction, resilience, school performance, high school students

1 Ministerio de Educación. Lima, Perú. 


\section{INTRODUCCIÓN}

El mundo actual ha cambiado y en cuanto a las formas de incorporar y transmitir conocimientos, gran parte de estos cambios se deben a las Tecnologías de la Información y de la Comunicación (TIC) optimizadas por el uso de Internet y las redes sociales (Cabero \& Llorente, 2015). Sin embargo, el uso inapropiado y en exceso de internet y las redes sociales repercute negativamente en adolescentes y jóvenes; diversos autores sugieren la presencia de una conducta adictiva que altera el estado emocional, las creencias y conductas en los adolescentes (Echeburúa \& del Corral, 2010; Instituto Nacional de Estadística e Informática [INEI], 2019). La región de Ucayali es la segunda región del país que cuenta con un mayor porcentaje de estudiantes con retraso escolar de más de 2 años llegando al 12,3\% del total de estudiantes (Ministerio de Educación [MINEDU], 2017) y el nivel secundario reúne la mayor cantidad de dichos estudiantes. Sobre el acceso a internet solo está disponible en el $38.1 \%$ de las instituciones educativas de esta región, siendo el más bajo a nivel nacional. Las cifras de adolescentes entre 14 y 19 años que no estudian ni trabajan según cifras del INEI (2018) durante el último censo nacional ascendió a $15,1 \%$ (varones $=12,2 \%$ y mujeres $=18 \%$ ), siendo la región Ucayali la tercera región del Perú que concentra mayores cifras en este aspecto, con un 5,6\% superior al promedio nacional. Los resultados de la Evaluación Censal de Estudiantes, ECE, del 2019, se presentan para la región Ucayali en un nivel por debajo del promedio nacional: de los estudiantes evaluados, sólo el 4,7\% en Matemática, 4,8\% en Lectura y 3,7\% en Ciencia y Tecnología, logran un nivel de desempeño satisfactorio (MINEDU, 2020).

La adicción a internet es definida como una conducta compulsiva (Matalinares et al., 2013), un uso excesivo de conexión a la red que conlleva síntomas como ansiedad, irritabilidad, desajustes emocionales y problemas en la socialización y que, a su vez, afecta las relaciones personales, familiares, al igual que al rendimiento escolar. La adicción a internet está compuesta por cuatro elementos: uso excesivo, retirada o abstinencia, tolerancia y consecuencias negativas (Matalinares et al., 2013). Estudios internacionales señalan como un predictor importante de la adicción a internet, la depresión y los rasgos de personalidad (Przepiorka et al., 2019). Un estudio en China detectó que los vínculos de amistad con "compañeros problema" mediaban, de forma parcial, la asociación inversa entre funcionamiento familiar y adicción a internet (Li et al., 2018). En el
Perú, Romo (2018) destacó que la adicción a internet se asocia inversamente a las habilidades sociales. Y el estudio de Ticona (2019), también en el Perú, reveló que el $28 \%$ de adolescentes presentó una conducta adictiva hacia internet, destacando que muchos padres desconocen el tiempo que sus hijos están conectados o que dedican a usar esta tecnología.

Un factor protector importante frente a la conducta adictiva es la resiliencia (Alonso et al., 2016; Pandian \& Lakshmana, 2017), la cual es entendida como un rasgo de personalidad relativamente estable y compuesto por elementos interrelacionados (Shaikh \& Kauppi, 2010), entre los que destacan el ser ecuánime, perseverante, auto confiado, satisfecho personalmente y tolerante a la soledad existencial (Wagnild \& Young, 1993). Un ser humano resiliente tiene la capacidad de afronte ante la adversidad frente a su salud propia y/o ajena, logrando fortalecerse a pesar de dicha situación. Adicionalmente, ante una situación de adversidad, hay capacidad de fortalecer las relaciones interpersonales. Son diversos los factores que inciden en la resiliencia. En un estudio con adolescentes de la selva peruana desarrollado por Sosa y Salas-Blas (2020) se observó una ausencia de diferencias según sexo en cuanto a resiliencia. Sanders et al. (2017) hallaron en adolescentes norteamericanos que los riesgos familiares $\mathrm{y}$ del vecindario afectan negativamente la resiliencia. Sin embargo, estos autores destacaron también que contar con una relación íntima con alguien y tener expectativas de educación promueven la conducta resiliente.

Por otro lado, la literatura destaca diversos resultados que indican el impacto de la resiliencia sobre aspectos de ajuste y salud mental. Por ejemplo, un estudio en Ecuador indica que la resiliencia influye en la calidad de vida y es predictor importante sobre la salud mental en adolescentes (Simón-Saiz et al., 2018). Además, la resiliencia parece ser un factor protector de la adicción a internet que difiere según el sexo (Nam et al., 2018), este resultado sugiere centrarse en los efectos de vulnerabilidad presentes en las mujeres quienes están más tiempo en redes sociales mientras los varones invierten su tiempo en juegos en línea.

El rendimiento escolar es conceptuado como la calificación cuantitativa y/o cualitativa, representada por la nota que, al ser coherente a través del tiempo, reflejará la adquisición de conocimientos, vale decir, el logro de los objetivos del programa preestablecido (Tournon, 1984, citado en Montero et al., 2007). Según 
Ferragut y Fierro (2012), anteriormente el rendimiento escolar estaba relacionado con la capacidad intelectual; sin embargo, actualmente el equilibrio personal resulta ser un elemento importante del logro de un mejor rendimiento. La literatura reporta el impacto de una serie de factores sobre el rendimiento académico de los escolares (e. g., Montero et al., 2007; Suárez et al., 2016). Según Montero et al. (2007), los factores que afectan el rendimiento escolar se agrupan en factores institucionales (infraestructura, número de estudiantes, acceso a servicios, etc.), factores pedagógicos (rol del docente, actitudes promovidas en los estudiantes), factores psicosociales (clima escolar percibido, personalidad del docente, etc.), y factores sociodemográficos (nivel educativo de los padres, sexo y procedencia del alumno, etc.). Un estudio previo realizado por García-Marín y Cantón-Mayo (2019), en adolescentes españoles, encontró diferencias en el rendimiento escolar según género: las mujeres lograron mejores puntajes en lingüística y ciencias, mientras que los varones en matemática. Los reportes de PISA (Programa Internacional para la Evaluación de Estudiantes) destacan la presencia de un mejor rendimiento en comunicación para las mujeres y para los varones en matemática (Bausela, 2016).

El estudio de Zhang et al. (2018) detectó que la relación entre rendimiento académico y adicción a internet estuvo mediada por la desmotivación académica y el compromiso académico, además hubo un rol moderador del promedio del rendimiento del aula, específicamente en aulas de alto rendimiento académico, que fue indistinto del sexo y edad del estudiante y del tamaño de aula. En el Perú, un estudio de Bedriñana (2019) destacó la relación significativa entre la resiliencia y el rendimiento académico en adolescentes de cuarto y quinto grado de secundaria. Otro estudio realizado en el Perú obtuvo resultados similares (Huaire, 2014).

La presente investigación tiene importancia teórica al indagar acerca del grado en que puede haber relación entre la adicción a internet, la resiliencia y el rendimiento académico en adolescentes de instituciones educativas públicas en una región con características sociales y educativas como las de Ucayali, en el Perú. Tal estudio también será de importancia práctica para tomar medidas preventivas y promocionales a fin de garantizar la continuidad educativa, el logro de los aprendizajes y una óptima salud psicológica en los estudiantes involucrados. Además, busca comparar la adicción a internet, la resiliencia y el rendimiento escolar según el sexo y el grado escolar en dichos adolescentes.

\section{MATERIAL Y MÉTODOS}

\section{Diseño}

El presente es un estudio empírico, de estrategia asociativa, y emplea un diseño correlacional controlado, ya que se analizó la relación entre variables considerando el efecto de terceras variables; también tuvo un componente comparativo (Ato et al., 2013).

\section{Participantes}

La población fueron estudiantes de secundaria de colegios estatales de los distritos de Callería y Yarinacocha en la ciudad de Pucallpa (región Ucayali, Perú). La muestra fue seleccionada por muestreo no probabilístico, accesible a la investigadora (Otzen \& Manterola, 2017) y estuvo compuesta por 519 escolares del tercer, cuarto y quinto grado de secundaria de 14 a 18 años de ambos sexos $(M=15,49$, $D E=1,026)$. El 53,9\% de estudiantes fueron mujeres y $46,1 \%$, varones. Respecto a "Vive con...", la mayor parte de estudiantes refirió vivir con padre y madre $(52,6 \%)$, solamente con mamá $(21,8 \%)$, solamente con papá $(14,8 \%)$, mientras que un $10.8 \%$ señaló vivir con otro familiar. De otra parte, el tiempo dedicado a conectarse a internet fue mayoritariamente menor a 01 hora diaria $(56,6 \%)$, y solo un $1,7 \%$ dedicaba más de 10 horas diarias. La principal actividad desarrollada en internet fue "hacer las tareas de la escuela" $(73 \%)$, seguido de "interactuar con mis amigos" (18,7\%) y "jugar videojuegos" (6\%).

\section{Instrumentos}

Test de Adicción a Internet (TAI): Este instrumento fue diseñado originalmente por Young (1998) y fue adaptado al Perú por Matalinares et al. (2013). El test está conformado por 19 ítems con opciones de respuesta tipo Likert que van de $0=$ nunca a $5=$ siempre. Dicho instrumento evalúa el nivel de adicción a internet a nivel global y en cuatro dimensiones: retirada, tolerancia, uso excesivo y consecuencias negativas. Es un cuestionario dirigido a adolescentes entre 13 a 19 años y cuya aplicación puede llevarse a cabo de manera individual o grupal. En el presente estudio se obtuvo un alfa de Cronbach de .905 .

Escala de Resiliencia (ER): Esta escala fue diseñada en 1993 por Wagnild y Young y cuenta 
con una validación al contexto peruano por Novella (2002). La escala está compuesta de 25 ítems, los cuales se responden mediante una escala tipo Likert de 7 puntos ( $1=$ En desacuerdo hasta $7=$ De acuerdo). Este instrumento evalúa cinco dimensiones: ecuanimidad, sentirse bien solo, confianza en sí mismo, perseverancia, y satisfacción y además proporciona una puntuación total. Dentro del presente estudio se obtuvo un valor alfa de Cronbach de .844.

Rendimiento escolar. Esta variable fue medida mediante una revisión documental de los registros de notas de los estudiantes, de ahí se extrajo el promedio de las calificaciones durante el cierre del año escolar 2017, las que fueron proporcionadas por la institución educativa en el 2018, de todos los escolares participantes del estudio.

Ficha de datos sociodemográficos: Esta ficha incluyó preguntas sobre el sexo, edad, grado, nombre de institución educativa, con quién vive el estudiante adolescente, principal lugar de acceso y el tiempo dedicado a internet y las actividades más desarrolladas dentro de este espacio virtual.

\section{Procedimiento}

La responsable del estudio realizó las coordinaciones y gestiones con cuatro instituciones educativas de Pucallpa. Una vez obtenidos los permisos, pudo la investigadora acceder y aplicar los instrumentos antes mencionados, además de tener accesibilidad a las calificaciones de los estudiantes. El recojo de datos fue en las aulas de clase, previo permiso del docente responsable y con la presentación a los estudiantes de motivo de la investigación y el consentimiento informado de las familias (padres, madres y/o apoderados), recalcando en éste la confidencialidad y anonimato de las respuestas.

\section{Análisis de datos}

Los datos fueron transcritos a un formulario diseñado en el programa Excel y después fueron exportados al programa SPSS versión 25 (IBM, 2017) y JASP versión 0.13 (JASP Team, 2020). En dichos programas se codificaron las variables de estudio para su correcta identificación y posterior análisis. Dentro del análisis inferencial, previamente de analizó el ajuste de las distribuciones de las variables (resiliencia y adicción a internet), por medio de la prueba de KolmogorovSmirnov. Dicha prueba arrojó una distribución sin normalidad de todas variables de estudio, por lo que se hizo uso de las siguientes pruebas estadísticas no paramétricas en la obtención de los resultados: $U$ de Mann Whitney, Kruskall Wallis y rho de Spearman. Siguiendo las normas de la APA (2020), los resultados estadísticos fueron acompañados por los estimadores del tamaño del efecto $\left(r^{2}, r_{b i s}\right.$ y $\left.\eta_{H}^{2}\right)$ a los que se aplicaron los criterios de interpretación de Cohen (1992), Domínguez-Lara (2017) y Ferguson (2009). Finalmente, para asegurar la calidad en los resultados se analizó la confiabilidad de las puntuaciones de los instrumentos utilizados.

\section{RESULTADOS}

La correlación entre resiliencia y adicción a internet fue baja y positiva $(r=.105)$. Al controlarse la variable rendimiento escolar, no se aprecian cambios en la relación entre ambas variables tal como se muestra en la tabla 1. Estas bajas correlaciones se reflejan en un tamaño de efecto que en todas las asociaciones fue insignificante $\left(r^{2}\right.$ entre .01 y .10 y $r$ menor a .20 , valor mínimo necesario para considerar significancia práctica).

De otra parte, hubo una correlación significativa entre resiliencia y promedio ponderado $(r=0,093)$ con un tamaño del efecto insignificante $\left(r^{2}\right.$ menor a 0,01 y $r_{s}$ menor a 0,20 ; véase tabla 2 ). No hubo correlación significativa de resiliencia con promedio de matemática y promedio de comunicación. Cuando fue controlada la variable adicción a internet, las relaciones no sufrieron cambios sustanciales, excepto en el caso de la relación entre resiliencia y promedio ponderado que pasó a mostrar un tamaño de efecto pequeño.

La tabla 3 presenta las correlaciones entre adicción a internet y rendimiento. Se aprecia una relación significativa baja y negativa entre la adicción a

Tabla 1. Relación entre resiliencia y adicción a internet, sin y con el rendimiento escolar (promedio de comunicación y promedio de matemática) como variable control, estudiantes de secundaria de la ciudad de Pucallpa $(\mathrm{n}=519)$

\begin{tabular}{|c|c|c|c|c|c|}
\hline & & Resiliencia & Resiliencia $^{(a)}$ & Resiliencia $^{(\mathbf{b})}$ & Resiliencia $^{(\mathrm{c})}$ \\
\hline \multirow{2}{*}{ TAI } & Correlación (sig.) & $0,105(0,017)$ & $0,118(0,007)$ & $0,108(0,014)$ & $0,116(0,008)$ \\
\hline & $r^{2}$ & 0,011 & 0,014 & 0,012 & 0,013 \\
\hline
\end{tabular}


Tabla 2. Correlación entre la resiliencia y rendimiento escolar (promedio de comunicación, promedio de matemática y promedio ponderado), sin y con la adicción al Internet como variable control, en estudiantes de secundaria de la ciudad de Pucallpa $(\mathrm{n}=519)$

\begin{tabular}{|c|c|c|c|c|}
\hline & & $\begin{array}{l}\text { Promedio } \\
\text { matemática }\end{array}$ & $\begin{array}{l}\text { Promedio } \\
\text { comunicación }\end{array}$ & $\begin{array}{l}\text { Promedio } \\
\text { ponderado }\end{array}$ \\
\hline & & \multicolumn{3}{|c|}{ Sin variable adicción a internet controlada } \\
\hline \multirow{5}{*}{ Resiliencia } & Correlación (sig.) & $0,058(0,189)$ & $-0,024(0,588)$ & $0,093(0,035)$ \\
\hline & $r^{2}$ & 0,003 & 0,001 & 0,009 \\
\hline & & \multicolumn{3}{|c|}{ Con variable adicción a internet controlada } \\
\hline & Correlación parcial (sig.) & $0,062(0,160)$ & $-0,014(0,748)$ & $0,106(0,016)$ \\
\hline & $r^{2}$ & 0,004 & $<0.001$ & 0,011 \\
\hline
\end{tabular}

Tabla 3. Correlación entre la adicción a internet y rendimiento escolar (promedio de comunicación, promedio de matemática y promedio ponderado), sin y con la resiliencia como variable control, en estudiantes de secundaria de la ciudad de Pucallpa $(n=519)$

\begin{tabular}{|c|c|c|c|c|}
\hline & & $\begin{array}{l}\text { Promedio } \\
\text { comunicación }\end{array}$ & $\begin{array}{l}\text { Promedio } \\
\text { matemática }\end{array}$ & $\begin{array}{l}\text { Promedio } \\
\text { ponderado }\end{array}$ \\
\hline & & \multicolumn{3}{|c|}{ Sin variable resiliencia controlada } \\
\hline & Correlación (sig.) & $-0,123(0,005)$ & $-0,149(0,001)$ & $-0,053(0,232)$ \\
\hline & $r^{2}$ & 0,015 & 0,022 & 0,003 \\
\hline \multirow[t]{3}{*}{ TAI } & & \multicolumn{3}{|c|}{ Con variable resiliencia controlada } \\
\hline & Correlación parcial (sig.) & $-0,107(<0.001)$ & $-0,156(<0.001)$ & $-0,068(0,125)$ \\
\hline & $r^{2}$ & 0,011 & 0,024 & 0,005 \\
\hline
\end{tabular}

Tabla 4. Comparación de las variables adicción a internet, resiliencia y rendimiento escolar (promedio comunicación, promedio matemática y promedio ponderado), según sexo, en estudiantes de secundaria de la ciudad de Pucallpa $(n=519)$

\begin{tabular}{|c|c|c|c|c|}
\hline \multirow[b]{2}{*}{ Variable } & \multicolumn{4}{|c|}{ Rango promedio } \\
\hline & $\begin{array}{l}\text { Varones } \\
(n=239)\end{array}$ & $\begin{array}{l}\text { Mujeres } \\
(n=280)\end{array}$ & U de Mann-Whitney (Z) & $r_{b i s}$ \\
\hline Resiliencia & 261,84 & 258,43 & $33020,5(-0,258)$ & $-0,011$ \\
\hline Confianza en sí mismo & 259,71 & 260,25 & $33390(-0,041)$ & $-0,002$ \\
\hline Ecuanimidad & 264,89 & 255,83 & $32291(-0,688)$ & $-0,030$ \\
\hline Perseverancia & 263,91 & 256,66 & $32526(-0,549)$ & $-0,024$ \\
\hline Satisfacción personal & 256,89 & 262,66 & $32716,5(-0,438)$ & $-0,019$ \\
\hline Sentirse bien solo & 258,53 & 261,26 & $33108,5(-0,207)$ & $-0,009$ \\
\hline Adicción a Internet & 251,63 & 267,15 & $31458,5(-1,176)$ & $-0,052$ \\
\hline Tolerancia & 251,17 & 267,54 & $31349,5(-1,240)$ & $-0,054$ \\
\hline Uso excesivo & 246,00 & 271,95 & $30114,5(-1,971)$ & $-0,087$ \\
\hline Retirada & 256,01 & 263,41 & $32506(-0,567)$ & $-0,025$ \\
\hline Consecuencias negativas & 263,05 & 257,40 & $32731.5(-0,432)$ & $-0,019$ \\
\hline Promedio de matemática & 249,52 & 268,95 & $30955(-1,488)$ & $-0,065$ \\
\hline Promedio de comunicación & 237,55 & 279,16 & $28094.5(-3,186)^{* *}$ & $-0,140$ \\
\hline Promedio ponderado & 232,16 & 281,89 & $26813.5(-3,774)^{* *}$ & $-0,166$ \\
\hline
\end{tabular}


internet con el promedio de comunicación $(r=-0,123)$ y el promedio de matemática $(r=-0,149)$, ambas también con tamaños de efecto insignificantes. No hubo correlación significativa entre adicción a internet y promedio ponderado. Al controlarse la variable resiliencia, las correlaciones entre adicción a internet y rendimiento académico fueron sustancialmente las mismas.

De acuerdo a la tabla 4, hubo diferencias significativas entre sexos solamente en el promedio de comunicación y el promedio ponderado, en ambos casos con un tamaño de efecto pequeño $\left(r_{b i s}\right.$ entre .10 y .30). Es de destacar que estas diferencias fueron a favor de las estudiantes mujeres.

En la tabla 5, haciendo uso de la prueba no paramétrica Kruskall-Wallis, se reportan diferencias significativas en resiliencia y en promedio de matemáticas según grado. Cabe precisar que estas diferencias, en ambos casos, fueron a favor de los escolares del cuarto grado, la segunda puntuación más alta fue la del grupo de quinto grado. Sin embargo, en todos estos casos, el tamaño del efecto fue inferior al considerado el mínimo necesario para indicar significancia práctica (Ferguson, 2009).
Siguiendo la tabla 6, se aplicó la prueba no paramétrica Kruskall-Wallis y no se aprecian diferencias significativas según "con quién vive", excepto en la variable promedio de matemática y promedio ponderado, donde hubo diferencia en favor de aquellos colegiales que viven con otro familiar, seguido de aquellos que viven con ambos padres (padre y madre) y en uso excesivo, que fue menor en los que viven con otro familiar, seguido de los que viven con ambos padres. Sin embargo, sólo en el caso del promedio de matemática, el tamaño del efecto fue el mínimo necesario para indicar significancia práctica.

La tabla 7 presenta las comparaciones según sexo en la principal actividad desarrollada en internet. Tanto varones como mujeres manifiestan que su principal actividad es hacer las tareas de la escuela. Mientras el porcentaje de mujeres que menciona esta actividad como la principal es más alto que en el caso de los varones, se aprecia que los estudiantes varones tienden a usar más videojuegos con respecto a las estudiantes mujeres. El tamaño del efecto asociado fue de magnitud pequeña.

Tabla 5. Comparación de las variables resiliencia, adicción a internet y rendimiento escolar (promedio comunicación, promedio matemática y promedio ponderado), según grado, en estudiantes de secundaria de la ciudad de Pucallpa $(\mathrm{n}=519)$

\begin{tabular}{lccccc}
\hline \multicolumn{1}{c}{ Variable } & \multicolumn{2}{c}{ Rango promedio según grado } & & \\
& $\begin{array}{l}\text { Tercero } \\
(\mathbf{n = 1 4 2})\end{array}$ & $\begin{array}{c}\text { Cuarto } \\
(\mathbf{n = 2 1 9 )}\end{array}$ & $\begin{array}{c}\text { Quinto } \\
(\mathbf{n = 1 5 8})\end{array}$ & Chi-cuadrado (gl) & $\eta_{H}^{2}$ \\
\hline Resiliencia & 220,31 & 284,40 & 261,85 & $15,774(2)^{* *}$ & 0,03 \\
Confianza en sí mismo & 224,16 & 281,01 & 263,09 & $12,525(2)^{*}$ & 0,02 \\
Ecuanimidad & 236,77 & 276,25 & 258,35 & $6,027(2)^{* *}$ & 0,01 \\
Perseverancia & 227,03 & 280,87 & 260,71 & $11,142(2)^{*}$ & 0,02 \\
Satisfacción personal & 227,20 & 276,67 & 266,37 & $9,850(2)^{* *}$ & 0,02 \\
Sentirse bien solo & 234,46 & 273,39 & 264,40 & $6,048(2)^{* *}$ & 0,01 \\
Adicción a Internet & 273,09 & 250,40 & 261,54 & $1,996(2)$ & - \\
Tolerancia & 272,29 & 253,26 & 258,31 & $1,418(2)$ & - \\
Uso excesivo & 266,00 & 253,78 & 263,23 & $0,682(2)$ & - \\
Retirada & 273,48 & 248,03 & 264,47 & $2,745(2)$ & - \\
Consecuencias negativas & 270,71 & 246,61 & 268,93 & $3,085(2)$ & - \\
Promedio de matemática & 232,34 & 283,74 & 251,96 & $11,017(2)^{*}$ & 0,02 \\
Promedio de comunicación & 263,10 & 267,00 & 247,51 & $1,671(2)$ & - \\
Promedio ponderado & 241,04 & 268,31 & 262,32 & $2,977(2)$ & - \\
\hline
\end{tabular}

Nota: $g l=$ grados de libertad; $* * p<.01 ; * p<.05$ 
Tabla 6. Comparación de las variables resiliencia, adicción a internet y rendimiento escolar (promedio comunicación, promedio matemática y promedio ponderado), según "con quién vive", en estudiantes de secundaria de la ciudad de Pucallpa $(\mathrm{n}=519)$

\begin{tabular}{|c|c|c|c|c|c|c|}
\hline \multirow[b]{2}{*}{ Variable } & \multicolumn{4}{|c|}{ Rango promedio según "con quién vive" } & \multirow[b]{2}{*}{ Chi-cuadrado (gl) } & \multirow[b]{2}{*}{$\eta_{H}^{2}$} \\
\hline & $\begin{array}{l}\text { Padre y madre } \\
(n=273)\end{array}$ & $\begin{array}{l}\text { Solo padre } \\
(n=77)\end{array}$ & $\begin{array}{l}\text { Solo madre } \\
(n=113)\end{array}$ & $\begin{array}{l}\text { Otro familiar } \\
(n=56)\end{array}$ & & \\
\hline Resiliencia & 270,95 & 241,08 & 257,46 & 237,77 & $3,945(3)$ & - \\
\hline Confianza en sí mismo & 274,42 & 238,66 & 253,44 & 232,29 & $6,235(3)$ & - \\
\hline Ecuanimidad & 261,72 & 260,58 & 259,70 & 251,40 & $0,223(3)$ & - \\
\hline Perseverancia & 267,43 & 240,23 & 258,73 & 253,54 & $2,127(3)$ & - \\
\hline Satisfacción personal & 268,11 & 253,60 & 266,08 & 217,03 & $5,761(3)$ & - \\
\hline Sentirse bien solo & 258,52 & 246,54 & 268,82 & 267,90 & $1,204(3)$ & - \\
\hline Adicción al Internet & 249,05 & 278,75 & 284,19 & 238,78 & $6,722(3)$ & - \\
\hline Tolerancia & 252,22 & 274,05 & 278,09 & 242,10 & $3,858(3)$ & - \\
\hline Uso excesivo & 248,09 & 287,94 & 281,91 & 235,46 & $8,362(3)^{*}$ & 0,02 \\
\hline Retirada & 254,65 & 263,46 & 280,31 & 240,37 & $3,501(3)$ & - \\
\hline Consecuencias negativas & 248,43 & 265,04 & 285,65 & 257,71 & $5,119(3)$ & - \\
\hline Promedio de matemática & 279,84 & 246,72 & 208,39 & 285,66 & $20,872(3)^{* *}$ & 0,04 \\
\hline Promedio de comunicación & 272,20 & 249,22 & 235,34 & 265,11 & $5,444(3)$ & - \\
\hline Promedio ponderado & 274,16 & 251,48 & 224,59 & 264,13 & $9,016(3)^{*}$ & 0,02 \\
\hline
\end{tabular}

Nota: $g l=$ grados de libertad; ${ }^{* *} \mathrm{p}<0,01 ; * \mathrm{p}<0,05$

Tabla 7. Principal actividad desarrollada en internet según sexo en estudiantes de secundaria de la ciudad de Pucallpa $(n=519)$

\begin{tabular}{|c|c|c|c|c|c|}
\hline & \multirow{2}{*}{ Variable } & \multicolumn{2}{|c|}{$\operatorname{Sexo}^{(a)}$} & \multirow{2}{*}{ Chi-cuadrado (gl) } & \multirow{2}{*}{$V_{\text {de Cramer }}$} \\
\hline & & Varón & Mujer & & \\
\hline \multirow{4}{*}{$\begin{array}{l}\text { Principal actividad } \\
\text { desarrollada en el } \\
\text { Internet }\end{array}$} & Hacer las tareas de la escuela & $70,3 \%$ & $75,4 \%$ & \multirow{4}{*}{$12,758(3)^{* *}$} & \multirow{4}{*}{0,157} \\
\hline & Interactuar con mis amigos & $20,9 \%$ & $16,8 \%$ & & \\
\hline & Jugar videojuegos & $8,4 \%$ & $3,9 \%$ & & \\
\hline & Otro & $0,4 \%$ & $3,9 \%$ & & \\
\hline
\end{tabular}

Nota: $g l$, grados de libertad; $* * \mathrm{p}<0,01 ; V_{\text {de Cramer }}$ tamaño del efecto; ${ }^{\left({ }^{(a)}\right.}$ porcentajes establecidos por las columnas.

\section{DISCUSIÓN}

El presente estudio abarcó una muestra de 519 adolescentes de la ciudad de Pucallpa, región Ucayali (Perú). Esta región es considerada con niveles bajos en logros de aprendizaje (MINEDU, 2020) y presenta elevadas cifras de menores que no estudian ni trabajan (INEI, 2018), así como bajo acceso a internet desde las instituciones educativas (INEI, 2019). Todo ello indica altos niveles de vulnerabilidad en esta población. En ese sentido, el objetivo del estudio fue determinar en qué grado están relacionados la adicción a internet, la resiliencia y el rendimiento escolar en adolescentes de instituciones educativas públicas de eta ciudad.
Los resultados señalan la relación directa entre la adicción a internet y resiliencia, situación controversial frente a lo esperado teóricamente en tanto la adicción a internet es una conducta de riesgo hacia la salud mental y la resiliencia es más bien un factor protector frente a una conducta adictiva (Alonso et al., 2016; Pandian \& Lakshmana, 2017). Al controlarse la variable rendimiento escolar, la relación entre adicción a internet y resiliencia no se vio afectada, sugiriendo una influencia despreciable del rendimiento escolar en la relación de dichas variables.

Siguiendo el reporte de Sanders et al. (2017), la resiliencia logra desarrollarse en adolescentes que están 
expuestos a situaciones de riesgo y cuando cuentan con una relación cercana con alguien. En el caso de la presente muestra de estudio, gran parte de ellos señaló vivir con ambos padres (52,6\%), lo que podría indicar la presencia de un factor protector importante en este grupo. Por otro lado, el tiempo dedicado a internet en los adolescentes fue predominantemente inferior a una hora $(56,5 \%)$. Esto permite inferir una baja adicción a internet en una gran proporción de participantes. Sin embargo, se carece de datos precisos respecto de la dinámica familiar en el grupo estudiado, por lo que no es posible conocer el impacto de este factor en el nivel de resiliencia en dichos estudiantes. A partir de los resultados, se podría sugerir que, en este caso, tratándose de estudiantes provenientes de comunidades rodeadas de múltiples carencias, la permanencia de la conexión a internet puede ser en sí misma una fuente de soporte y, por tanto, de resiliencia. Sin embargo, los tamaños de efecto insignificantes observados permiten suponer también que la relación encontrada carece de significancia práctica.

Estudios con adolescentes encontraron relaciones directas entre la resiliencia y el rendimiento escolar (Bedriñana, 2019; Meza-Cueto et al., 2017), hallazgos que son consistentes sólo con el resultado referente al promedio ponderado en el presente estudio, en cuyo caso la relación mostró un tamaño de efecto pequeño sólo cuando se controló la variable de adicción a internet. La correlación no significativa con asignaturas específicas como son matemática y comunicación, suscita reflexiones en cuanto a la necesidad de que el rendimiento escolar debiera reforzar la evaluación por competencias sin reducirse a solo áreas temáticas tan delimitadas como las evaluadas por PISA a nivel internacional y UMC a nivel nacional (MINEDU, 2020).

Por otro lado, la relación entre la adicción a internet y el promedio ponderado no fue significativa, pero sí lo fue entre adicción y el rendimiento en matemática y en comunicación. Estos resultados sugieren que la adicción a internet estaría afectando negativamente el desempeño en asignaturas objeto de medición dentro de la ECE. Se puede considerar que estos resultados se encentran dentro de lo esperado. Sin embargo, en estos casos las correlaciones también indican tamaños de efecto insignificantes. Si consideramos solamente el aspecto de la significación estadística, se puede comentar que la falta de correlación entre adicción y el promedio ponderado podría estar indicando un menor impacto de la adicción en otras de las materias escolares evaluadas. Aparte de ello, las condiciones de uso de internet por los estudiantes encuestados podrían tener también algún impacto en el rendimiento. Se debe considerar que el uso de los recursos virtuales en las asignaturas es limitado debido a las condiciones estructurales de las instituciones educativas de la región Ucayali (MINEDU, 2017); por tanto, el acceso a internet es realizado principalmente fuera de la institución educativa, sin garantías de una adecuada orientación por parte del docente afectando el rendimiento escolar (Montero et al., 2007). En el presente estudio los adolescentes indicaron que la actividad principal desarrollada en internet fueron las actividades académicas (73\%), sin embargo, estas podrían estar siendo interrumpidas con lapsos de interacción con amigos y juegos online.

Las comparaciones de las tres variables según sexo mostraron diferencias significativas solo en la variable rendimiento escolar (promedio de comunicación y promedio ponderado), siendo las calificaciones más altas en mujeres. Esto concuerda con el hallazgo de Garcí-Marín y Cantón-Mayo (2019), estudio donde participaron 1488 adolescentes de 12 a 18 años de instituciones educativas estatales de España. Lo anterior refuerza las brechas de género identificada en mediciones internacionales como PISA donde las mujeres logran mejores calificaciones en comunicación $\mathrm{y}$, de forma inversa, los varones obtienen mayores calificaciones en matemáticas (Bausela, 2016). Lo reportado sobre la ausencia de diferencias de resiliencia según sexo coincide con el estudio de Sosa y SalasBlas (2020), quienes trabajaron con una muestra de adolescentes de la selva peruana.

Los adolescentes del presente estudio alcanzaron diferencias significativas en la variable resiliencia y sus dimensiones según el grado de estudios, siendo mayor en los adolescentes del cuarto de secundaria. Sin embargo, en todas esas puntuaciones, la magnitud del efecto fue menor al valor 0.04 considerado como el mínimo para establecer una diferencia con significancia práctica (Ferguson, 2009). La literatura señala la resiliencia como un indicador de la salud mental del adolescente (Simón-Saiz et al., 2018). La mayor o menor proximidad al termino de los estudios escolares podría ser considerado un factor de estrés en los adolescentes, por lo que los resultados parecen indicar un nivel más o menos equilibrado entre los tres grupos en las posibilidades de afrontamiento frente a esta circunstancia.

De acuerdo al tipo de cuidador principal del adolescente, se observó diferencias significativas en 
uso excesivo, el promedio de matemática y promedio ponderado. Se aprecia que, en cuanto al uso excesivo, el mayor puntaje fue en el grupo de vivir solo con el padre y el menor puntaje fue en vivir con ambos padres y con otro familiar. Asimismo, vivir con ambos padres parece incidir en un mejor rendimiento. Estos resultados reforzarían la importancia de un grupo familiar integrado como factor protector. La presencia de los padres juega un rol importante en la regulación del tiempo dedicado por los adolescentes a internet y en la supervisión de las actividades académicas, y ello tiene implicaciones en el desarrollo o no de la adicción a internet; por ejemplo, Ticona (2019) señaló que la adicción a internet en los adolescentes se asoció al desconocimiento de los padres sobre el tiempo de conexión a internet que tenían sus hijos. No obstante, nuevamente hay que considerar que la única comparación en que se observó un nivel mínimo de significancia práctica fue la correspondiente al promedio en matemática.

Finalmente, el presente estudio reporta que las actividades más desarrolladas por los adolescentes son "hacer las tareas de la escuela" e "interactuar con mis amigos", sin embargo, el "jugar videojuegos" fue más frecuente en los varones. Esto último coincide con los hallazgos de Nam et al. (2018), quienes destacan, además, distintos factores de riesgo para cada género, al señalar que las mujeres serían más vulnerables al permanecer más tiempo en redes sociales y contactar con desconocidos mientras los varones están más horas en juegos en línea. Una adecuada regulación por parte de los propios adolescentes y de sus padres en relación con el tiempo de conexión y el tipo de actividad a la que se dedican evitará el desarrollo de conductas compulsivas hacia internet.

La autora concluye en la presencia de algunas relaciones entre las variables estudiadas, aunque con tamaños de efecto pequeños. Se considera recomendable desarrollar estudios posteriores en adolescentes que están fuera del sistema educativo a fin de contrastar con los presentes hallazgos. Además, posiblemente variables como motivación y compromiso académico estén mediando la relación entre adicción a internet y rendimiento escolar (Zhang et al., 2018), por lo que su incorporación en futuros estudios sería de interés en la población de estudio. Una limitación del estudio fue trabajar con un muestreo no probabilístico lo que impide la generalización de los resultados a grupos de similares características. Los hallazgos quedan abiertos a discusión en futuros estudios.

\section{REFERENCIAS BIBLIOGRÁFICAS}

Alonso, M.M., Camacho, J.U., Armendáriz, N.A., Alonso, B.A., Ulloa, J.L., \& Pérez, S. (2016). Resiliencia y consumo de alcohol en adolescentes del municipio de Cunduacán, Tabasco. CIENCIA UANL, 19(79), 56-61. http://eprints.uanl.mx/10952/1/ Documento9.pdf

American Psychological Association (2020). Publication manual. (7ma. ed.). Autor.

Ato, M., López, J., \& Benavente, A. (2013). Un sistema de clasificación de los diseños de investigación en psicología. Anales de Psicología, 29(3), 1038-1059. http://doi.org/10.6018/ analesps.29.3.178511

Bausela, E. (2016). Análisis comparativo de la probabilidad de tener bajo rendimiento en función del sexo en las competencias PISA 2012. Revista Iberoamericana de Diagnóstico y Evaluación - e Avaliação Psicológica, 1(41), 58-65.

Bedriñana, A. (2019) Correlación entre la resiliencia y rendimiento académico en estudiantes de 4to y 5to de secundaria de la Institución Educativa Jean Piaget, Ayacucho. [Tesis de licenciatura. Universidad Federico Froebel]. Repositorio Institucional de la Universidad Federico Froebel. http://repositorio. udaff.edu.pe/bitstream/handle/20.500.11936/151/ INFORME \% 20 FINAL.pdf? sequence $=1$ \&isAllowed $=\mathrm{y}$

Cabero, J., \& Llorente, M. (2015). Tecnologías de la Información y la Comunicación (TIC): escenarios formativos y teorías del aprendizaje. Revista Lasallista de Investigación, 12(2), 186-193.

Cohen, J. (1992). A power primer. Psychological Bulletin, 112(1), 155-159. http://doi. org/10.1037//0033-2909.112.1.155

Domínguez-Lara, S. A. (2017). Magnitud del efecto, una guía rápida. Educación Médica, 19(4), 251-254. http://doi.org/10.1016/j.edumed.2017.07.002

Echeburúa, E., \& del Corral, P. (2010). Adicción a las nuevas tecnologías y a las redes sociales en jóvenes: un nuevo reto. Adicciones, 22(2), 91-96. http://dx.doi. org/10.20882/adicciones. 196

Ferguson, C. J. (2009). An effect size primer. A guide for clinicians and researchers. Professional Psychology: Research and Practice, 40(5), 532-538. http://doi.org/10.1037/a0015808

Ferragut, M., \& Fierro, A. (2012). Inteligencia emocional, bienestar personal y rendimiento académico en preadolescentes. Revista Latinoamericana de Psicología, 44(3), 94-105.

García-Marín, S., \& Cantón-Mayo, I. (2019). Uso de tecnologías y rendimiento académico en estudiantes adolescentes. Comunicar. Revista Cientifica de Educación y Comunicación, 27(59), 73-81. https:// doi.org/10.3916/C59-2019-07 
Huaire, E. (2014). Prevalencia de resiliencia y autoestima sobre el rendimiento escolar en estudiantes de instituciones educativas de Ate Vitarte, Lima. Apuntes de Ciencia \& Sociedad, 4(2), 202-209. http://doi. org/10.18259/acs.2014-023.

IBM Corp. Released (2017). IBM SPSS Statistics for Windows, Version 25.0. Armonk, NY: IBM Corp.

Instituto Nacional de Estadística e Informática (2018). Adolescentes que no estudian ni trabajan y su condición de vulnerabilidad. Instituto Nacional de Estadística e Informática https:/www.inei.gob.pe/ media/MenuRecursivo/publicaciones_digitales/Est/ Lib1632/libro.pdf

Instituto Nacional de Estadística e Informática (2019). Estadísticas de las Tecnologías de Información y Comunicación en los Hogares. Trimestre: OctubreNoviembre - diciembre 2018. Instituto Nacional de Estadística e Informática https:/www.inei.gob. pe/media/MenuRecursivo/boletines/01-informetecnico-n01_tecnologias-de-informacion-oct-novdic2019.pdf

JASP Team (2020). JASP (Version 0.13) [Computer software]. Amsterdam, University of Amsterdam.

Li, J., Li, D., Jia, J., Li, X., Wang, Y., \& Li, Y. (2018). Family functioning and internet addiction among adolescent males and females: A moderated mediation analysis. Children and Youth Services Review, 91, 289297. http://dx.doi.org/10.1016/j.childyouth.2018.06. 032

Matalinares, M., Raymundo, O., \& Baca, D. (2013). Propiedades psicométricas del test de adicción al internet (TAI). Revista Peruana de Psicología y Trabajo Social, 3(2), 45-66.

Meza-Cueto, L., Abuabara-Moya, N., Pontón-Villareal, D., Ortega-Mercado, F., \& Stave-Mendoza, F. (2020). Factores personales de la resiliencia y desempeño académico de escolares en contexto de vulnerabilidad. Búsqueda, 7(24), e491. http://doi. org/10.21892/01239813.491

Ministerio de Educación (2017). Presentación del proceso censal 2017 - MINEDU. Ministerio de Educación.http://escale.minedu.gob.pe/c/document library/get_file?uuid=d524d4b5-0dd3-4706-a1e8c65fb18a3d77\&groupId=10156

Ministerio de Educación (2020). Evaluaciones de logros de aprendizaje. Resultados 2019. Ministerio de Educación. http:/umc.minedu.gob.pe/wp-content/ uploads/2020/06/PPT-web-2019-15.06.19.pdf

Montero, E., Villalobos, J. \& Valverde, A. (2007). Factores institucionales, pedagógicos, psicosociales y sociodemográficos asociados al rendimiento académico en la Universidad de Costa Rica: Un análisis multinivel. RELIEVE, 13(2), 215-234. https:// doi.org/10.7203/relieve.13.2.4208

Nam, C.R., Lee, D.H., Lee, J.Y., Choi, A.R., Chung, S.J., Kim, D.J., Bhang, S.Y., Kwon, J.G., Kweon, Y.S.,
\& Choi, J.S. (2018). The role of resilience in internet addiction among adolescents between sexes: A moderated mediation model. Journal of Clinical Medicine, 7(8), 222. http://doi.org/10.3390 jcm 7080222

Novella, A. (2002). Incremento de la resiliencia luego de la aplicación de un programa de psicoterapia breve en madres adolescentes. [Tesis de maestría]. Universidad Nacional Mayor de San Marcos.

Otzen, T. \& Manterola, C. (2017). Técnicas de muestreo sobre una población a estudio. International Journal of Morphology, 35(1), 227-232. https://dx.doi. org/10.4067/S0717-95022017000100037

Pandian, R.D., \& Lakshmana, G. (2017). Risk and resilience factors for substance use among street adolescents: Assessment and development of an integrative model. Asian Social Work and Policy Review, 11(3), 216-233. http://doi.org/10.1111/ aswp. 12127

Przepiorka, A., Blachnio, A., \& Cudo, A. (2019). The role of depression, personality, and future time perspective in internet addiction in adolescents and emerging adults. Psychiatry Research. 272, 340-348. http://doi.org/10.1016/j.psychres.2018.12.086

Romo, D. (2018). Adicción a internet y habilidades sociales en estudiantes de secundaria de la institución educativa Internacional Elim de Villa El Salvador2017 .[Tesis de maestría. Universidad César Vallejo]. Repositorio Institucional de la Universidad César Vallejohttp://repositorio.ucv.edu.pe/handle/UCV/141 22?locale-attribute $=$ en

Sanders, J., Munford, R., \& Boden, J. (2017). Culture and context: The differential impact of culture, risks and resources on resilience among vulnerable adolescents. Children and Youth Services Review, 79, 517-526. http://doi.org/10.1016/j. childyouth.2017.07.007

Shaikh, A. \& Kauppi, C. (2010). Deconstructing resilience: myriad conceptualizations and interpretations. International Journal of Arts and Sciences, 3(15), 155-176. http://openaccesslibrary. org/images/TNT218_Carol_Kauppi.pdf

Simón-Saiz, M.J., Fuentes-Chacón, R.M., GarridoAbejar, M., Serrano-Parra, M.D., Larrañaga-Rubio, E., \& Yubero-Jiménez, S. (2018). Influence of resilience on health-related quality of life in adolescents. Enfermería Clínica, 28(5), 283-291. http://doi.org/10.1016/j.enfcli.2018.06.003

Sosa, S. S., \& Salas-Blas, E. (2020). Resiliencia y habilidades sociales en estudiantes secundarios de San Luis de Shuaro, La Merced. Comuni@cción, 11(1), 40-50. http://dx.doi.org/ 10.33595/22261478.11.1.394

Suárez, S., Elías, R. \& Zarza, D. (2016). Factores asociados al rendimiento académico de estudiantes de Paraguay: un análisis de los resultados del TERCE. 
Revista Iberoamericana sobre Calidad, Eficacia y Cambio en Educación-REICE, 14(4), 113-133. https://doi.org/10.15366/reice2016.14.4.006

Ticona, M. (2019). Factores de riesgo asociado a la conducta de adicción a internet en adolescentes, institución educativa secundaría Independencia Nacional, Puno - 2016 [Tesis de licenciatura. Universidad Nacional del Altiplano].Repositorio Institucional de la Universidad Universidad Nacional del Altiplano. http://repositorio.unap.edu.pe/ bitstream/handle/UNAP/11726/Ticona_Rodriguez_ Martha.pdf?sequence $=1 \&$ isAllowed $=\mathrm{y}$

Wagnild, G.M. \& Young, H. M. (1993). Development and psychometric evaluation of the Resilience Scale. Journal of Nursing Measurement, 1(2), 165178.
Young, K. (1998). Caught in the Net. John Wiley \& Sons. Inc.

Zhang, Y., Qin, X., \& Ren, P. (2018). Adolescents' academic engagement mediates the association between Internet addiction and academic achievement: The moderating effect of classroom achievement norm. Computers in Human Behavior, 89,299-307.http://doi.org/10.1016/j.chb.2018.08.018

\section{Correspondencia:}

Elsa M. Sánchez-Yarmas

Correo electrónico: elsanchez@minedu.gob.pe

Recibido: 16/01/2021

Aceptado: 12/05/2021 